\title{
Automation Lecture Scheduling Information Services through the Email Auto-Reply Application
}

\author{
Syahrul Mauluddin ${ }^{1}$ \\ Department of Informatics Management \\ Universitas Komputer Indonesia \\ Bandung, Indonesia
}

\author{
Leonardi Paris Hasugian ${ }^{2}$, Andri Sahata Sitanggang ${ }^{3}$ \\ Department of Information System \\ Universitas Komputer Indonesia \\ Bandung, Indonesia
}

\begin{abstract}
The study program of information systems is one of the largest studies programs at Indonesian Computer University (UNIKOM). In the process of scheduling lectures in the study program of information systems, it has already information systems of used desktop-based lecture scheduling. Lecture schedules that have been created are then informed through various media such as trust online, social media, email and bulletin board. With so many media which are used in the delivery of lecturers schedule it is expected that lecturers, students and laboratory staff can obtain schedule information properly. However, this also frequently causes problems in learning activities like misplaced of room, time, class, and so on. This usually occurs because the schedule in one of the communication media about lecture schedule is not updated when there is a change of schedule, so there are differences in the schedule information among lectures, students and laboratory staff. To overcome these problems, it needs a service center of lecture schedules information to facilitate lecturers, students and laboratory staff in obtaining the latest lecture schedules information. Related to this, in this study we propose a design of email auto-reply application that will be the service center of lecturer schedules information. In this study, the research method is the method of object-oriented approach and the method of prototype system development. In building email autoreply application, we are using the Java programming language with MySQL database. With the applications, it is expected that lecturers, students and laboratory staff can obtain the latest lecture schedule easily and from the same source, so different lecture schedules among lectures, students and laboratory staff do not happen again.
\end{abstract}

Keywords-Email; auto-reply; service center; lecture schedule; java programming

\section{INTRODUCTION}

The lectures scheduling process is one activity that is very important in the academic activities of a college. A good academic activity should be supported by a good lecture scheduling system starting from the process of scheduling until the deployment of schedule information that has been made so that academic activities can be run according to the academic calendar which has been specified.

The study program of information system is one of the largest studies programs at Indonesian Computer University. In the process of lecture scheduling in the study program of information systems, it has been already using the desktopbased information system of lecture schedule that can help to overcome the problems of conflicting schedules. The lecture schedules that have been created are then informed through various media such as trust online, social media, email and bulletin board. With so many media which is used in the delivery of schedule information, it is expected that all lecturers and students can get timetable well. However, this is also frequent to cause any problems in the lecturing process.

In the lecture scheduling, there are still common problems, such as the wrong room, wrong time, wrong class, and so on. The problems usually occur because of the schedule information in one of the communication media that the schedule is not updated when there is a change of schedule, resulting in differences in the schedule information between lecturers and students. If there is a scheduling problem usually students meet the secretariat of the study program to ask for the phone number of lecturers and also ask for the latest schedule. To overcome these problems, it needs service center of lecture schedule information to facilitate lecturers, students and laboratory staff in obtaining the latest lecture schedule information.

Based on the evaluation result of the methods of lectures schedule information dissemination that are running and the evaluation of information system of lecture scheduling application, then to meet these needs, it is proposed to create an application that will be the information service center of lecture schedule in the form of email auto-reply applications that will send lecture schedule information automatically according to the request which is sent via email.

With the above application, it is expected to lecturers, students and laboratory staff can obtain lecture schedule information easily without having to visit the secretariat and obtain lecture schedule information from the same source so that it does not happen anymore the schedule difference between lecturers, students and laboratory staff.

\section{LITERATURE REVIEW}

\section{A. Application}

The application is "software that is created to work on solving specific problems" [1].

\section{B. Email}

"Electronic mail (Email) is a letter which is delivered through an electronic device that is called a computer. To send and to access email on the mail server is required protocol "[2]. 


\section{Simple Mail Transport Protocol (SMTP)}

Simple Mail Transport Protocol (SMTP) is "used to communicate and send emails to server. This protocol function is only focused as the email sender to server. It does not function as the email recipient of the server. So, SMTP could not find any message in the mailbox or create an email in the email application directory. So, this protocol is known as Mail Transfer Agent (MTA). This protocol runs on port 25 by its default"[2].

\section{Post Office Protocol Version 3 (POP3)}

"Version 3 of the Post Office Protocol (POP3) is comparatively simple, and only allows the user to download emails from the server to the client. The user can $\log$ in to an account, view the contents of the mailbox, transfer and delete emails, and log out, all via server port 110 . This requires few resources, and there is little to configure, which means few sources of error. Emails are stored locally on the user's PC, which saves precious storage space on the server and reduces backup times. The user usually has to download all emails before deciding which ones are worth reading, based on the subject and/or the sender, although by now most clients support filters for screening incoming mail messages"[3].

\section{E. Internet Message Access Protocol (IMAP)}

"It is the development of POP3 protocol which is the system works is more complex. This protocol works when the mail client user connects to the mail server and successfully verify based on the user and password. Then users read the email that come that is when IMAP protocol works, this protocol that visualizes to users the email which were read by the users of the email client"[2].

\section{F. Email Gateway}

"Email Gateway is an application-based functionality that allows someone to start a script by sending an email message to the address that we want to go. This application can automatically process email messages from customers and provide feedback process by sending an email notification. Email Gateway acts as an SMTP email recipient. However, in addition to storing or forwarding received email messages, email gateway can specify the script that should be used to process email messages. The script is used to receive and process the content of email messages or to respond to email messages, logs, and to create automatic error handling procedures, which can be changed in accordance with the user wishes. By using an email gateway, transaction reports can be sent automatically to the email administrator" [4].

\section{G. Unified Modelling Language}

"Unified Modeling Language (UML) is a family of graphical notation that is supported by single meta-model, which enables the description and design of software systems, especially systems which are built using object-oriented programming $(\mathrm{OO})$. UML is a relatively open standard that is controlled by Object Management Company (OMG), an open consortium that consists of many companies"[5].

\section{H. Use Case Diagram}

"Use Case is a technique to record the functional requirements of a system. Use Case describes a typical interaction among the system users with the system itself, by giving a narrative of how the system is used. Use Case diagram shows which the actors are using use case that includes another use case and the relation between actor and use case"[5].

\section{Activity Diagram}

"Activity diagram is a technique to describe procedural logic, business processes, and work flow. In some cases, activity diagram plays a role which is similar to flowcharts, but the principle difference is between flow diagram notations that activity diagram supports behavior parallel. Node on an activity diagram is called the action, so the diagram displays an activity that is composed of action"'[5].

\section{J. Sequence Diagram}

"A sequence diagram is a dynamic diagram that shows what happens during the time. In this diagram, all the details of the operations are specified, and the messages that each object involved in the operation sends to the others is detailed together with the time instant at which it happens. A sequence diagram is a time-based representation on messages in the system because it shows time line of events that happen in the system. Sequence diagram shows what happens when a particular flow through a use case or activity diagram is executed. It also shows set of collaborating objects, in addition to showing the messages that pass between them"[6].

\section{K. Lecture Schedule}

The definition of lecture schedule refers to the definition of learning activities scheduling according to Tomhart. "Scheduling of teaching and learning activities is arrangements of teaching and learning planning which include subjects, lecturers, time and place at university. In general schedule of teaching and learning activities are presented in the days of the week table which consists of the time slot. It is comprised of subjects, days, hours, and teachers in accordance with the teaching subjects"[7].

\section{RESEARCH METHOD}

\section{A. Data Collecting Method}

The data collected in this study came from two sources as follows:

1) Primary data sources: The data is derived from primary data source which is obtained by using two ways:

\section{a) Observation}

Observation is a data collection technique through direct observation of symptoms or events that occur on the research object. In this case writer made some observations to observe the physical state, the location or place of study, namely Studies Program of Information Systems at Indonesian Computer University.

\section{b) Interview}

Interview is data collection technique through face to face and direct discussion between data collector (writer) with parties related to the research object. In this case interviews are conducted by secretary and secretariat of information systems study program. 
2) Secondary data sources: The data are derived from secondary data sources that are obtained by documentation technique. Documentation is data collection techniques by collecting documents which are related to the research object. In this study, the necessary documents are Lecture Schedule, Minutes of Class, Lecturers' and Students' Phonebook as well as Class Use Table.

3) Research methods: In this study the research method which is used is the method of object-oriented approach. "Object-oriented analysis and design can offer an approach that facilitates logical, rapid, and thorough methods for creating new systems responsive to a changing business landscape" [8]. Method of system development, it is a prototype model. The steps of prototype models can be seen in Fig. 1.

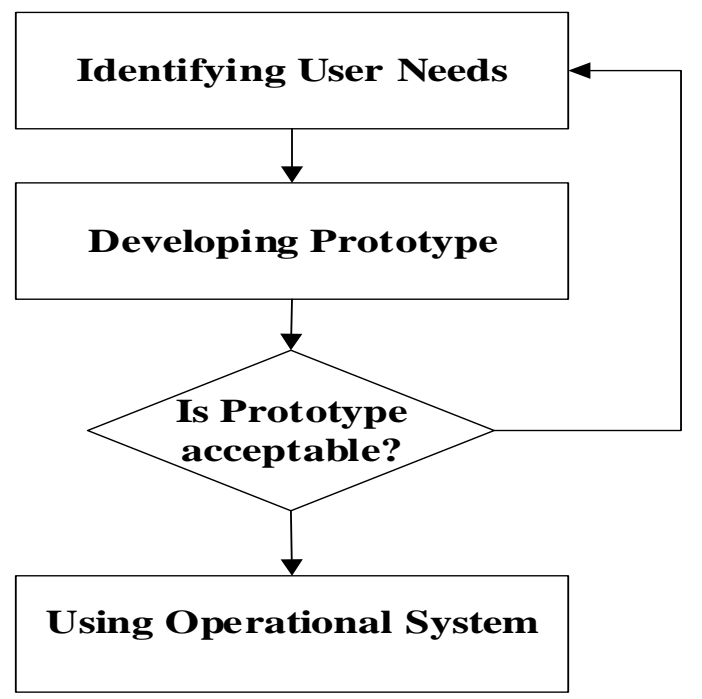

Fig 1. Prototype Model [9].

Here is the explanation of each step of the prototype models:

1) Identifying the user needs. At this step, we interviewed users to get an idea of what it is wanted by the user to the system/application.

2) Developing Prototype. At this step, we do the designing of email auto-reply application with conducting the applications functionality designing, database designing, interface application designing, format designing of email delivery.
3) Determining whether the prototype is acceptable. Users provide input to the analyst whether the prototype is already as the needs or not. If it is not then going back to an earlier step.

\section{RESULT}

This section will explain the steps which are undertaken in designing email auto-reply applications and explain the achievements of each steps. The following steps refer to the prototype model system development methods.

\section{A. Identifying User Needs}

The end result of this first step is known that study program of information system requires the service center of lecture schedule information to facilitate all parties who are interested in the lectures schedule.

Based on the evaluation results to the methods of information dissemination on lectures' schedules that are running and the evaluation of lectures scheduling information system application, so to meet the needs of information study program it is proposed to create an application that will be the central of information service to lectures schedule in the form of email auto-reply applications which will send lecture schedule information automatically according to the request which is sent via email. Several reasons make email as a request media and information delivery of lectures schedule as follows: 1) All lecturers, students and laboratory staff have email as it is facilitated by university. 2). The use of email is easier with the email's application on smartphones, tablets and ipad. 3). Email is capable of sending large file size for sending the schedule file in the form of .pdf and .xls files.

\section{B. Developing Prototype}

At the step of developing this prototype it will explain the results of the applications functionality design, database design and email delivery format design.

1) Draft of application functionality: At the design step, it will be explained the draft of application functionality is through use case diagram and it will be explained the overview of user activity to joint application on system reaction through activity diagram.

\section{a) Use Case Diagram of Email Auto-reply Application}

The functionality of email auto-reply application consists of two that can be seen in Fig. 2.

b) Activity Diagram of Email Auto-reply Applications

The flow picture of process that occurs in each use case can be seen in Fig. 3 and 4. 


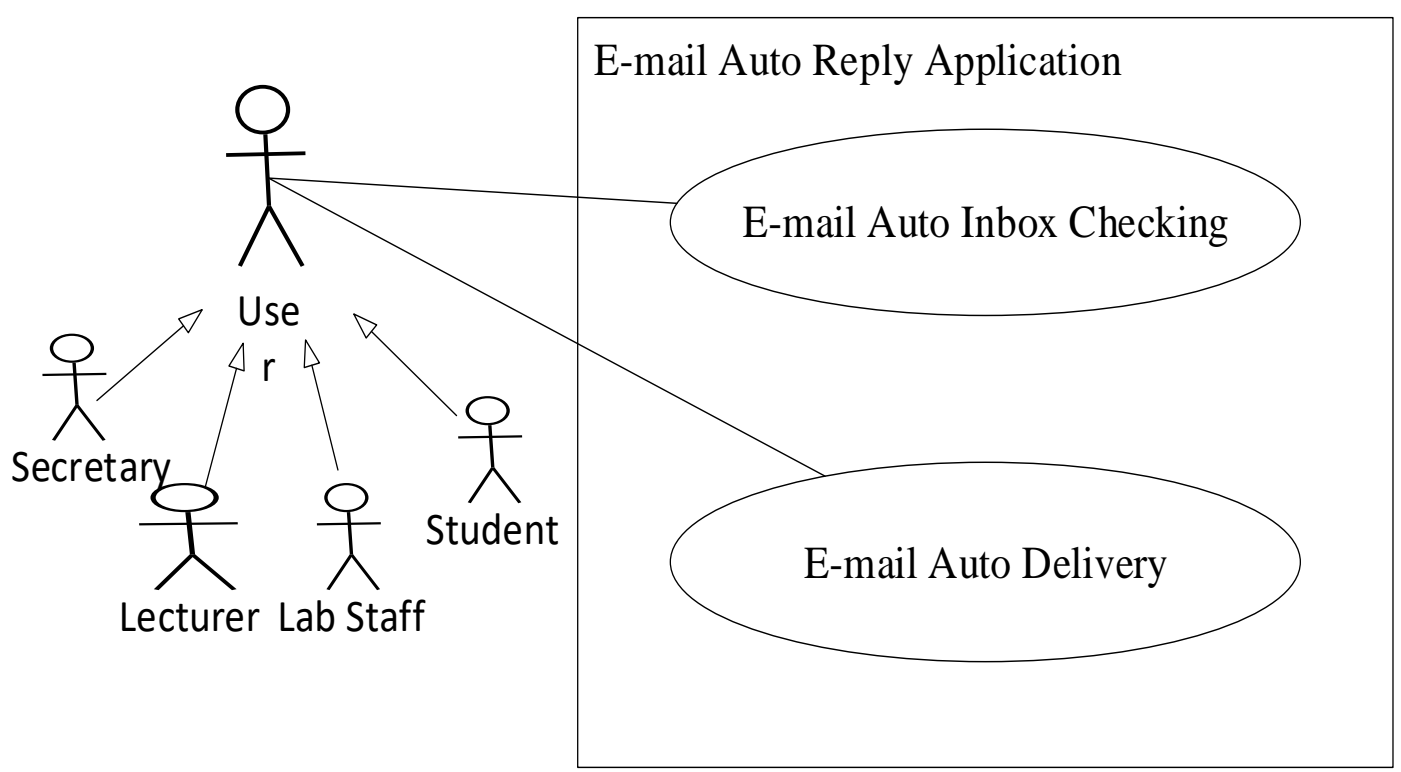

Fig 2. Use Case Diagram of Email Auto-Reply Application.

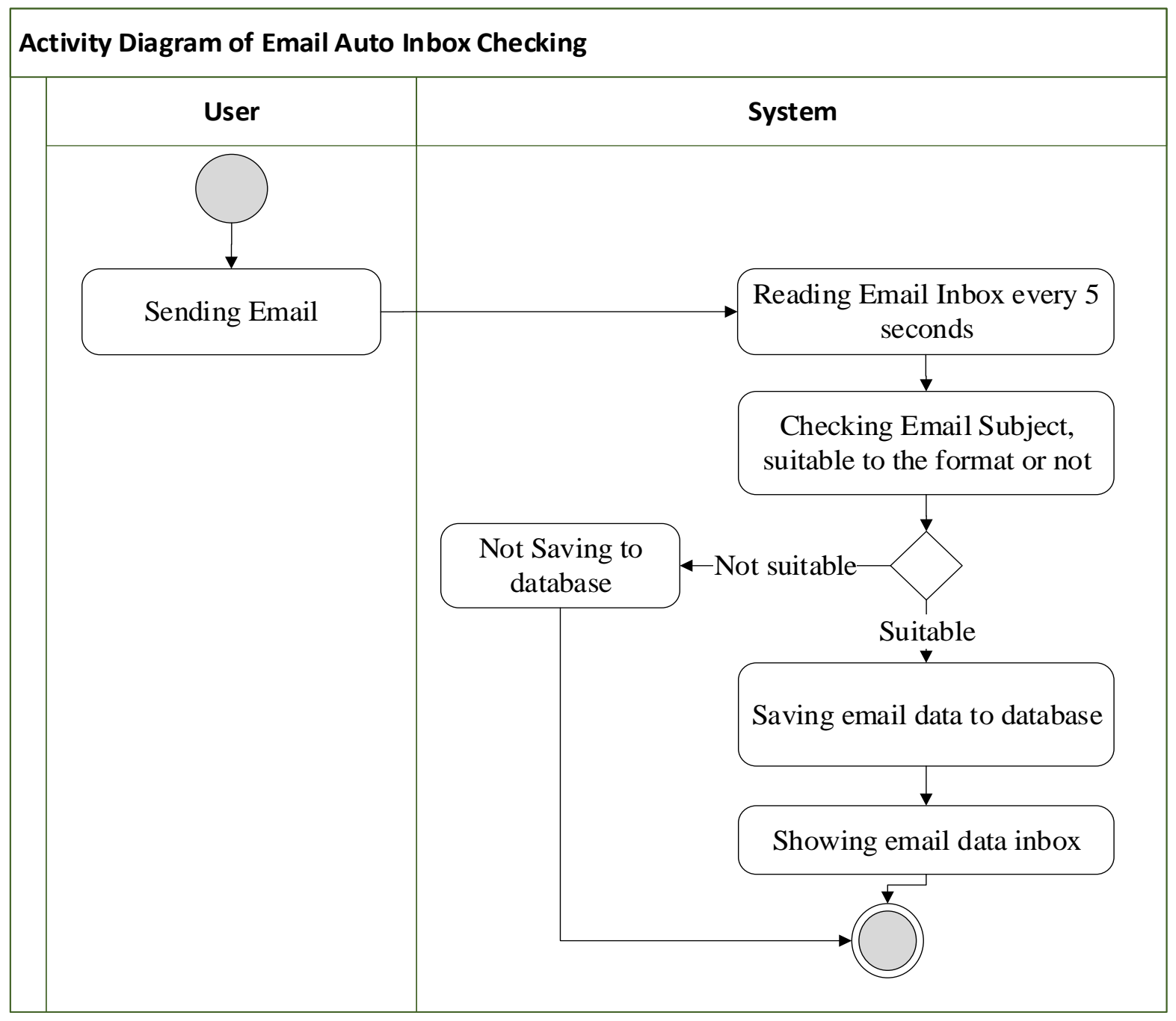

Fig 3. Activity Diagram of Email Auto Inbox Checking. 


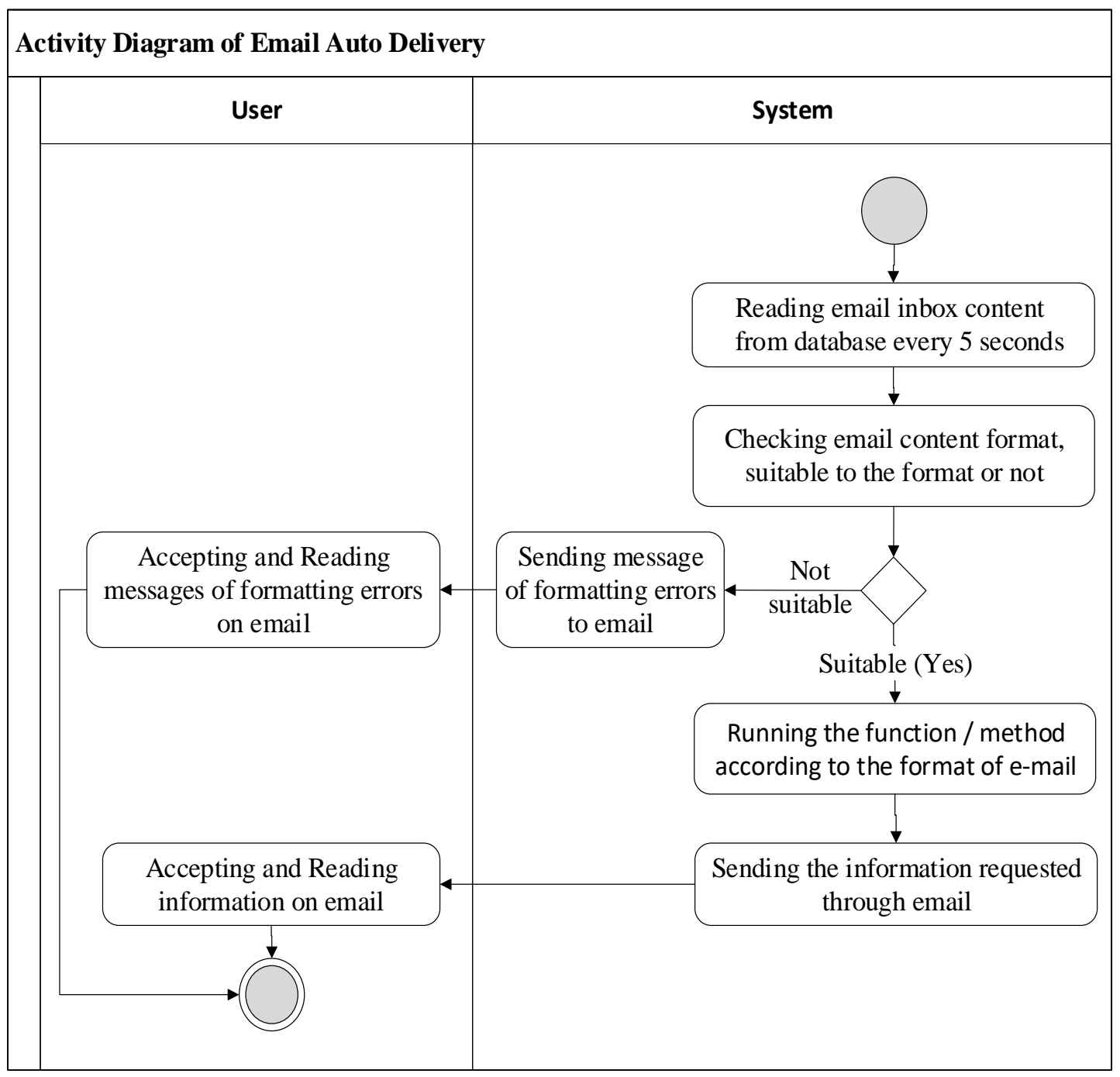

Fig 4. Activity Diagram of Email Auto Delivery.

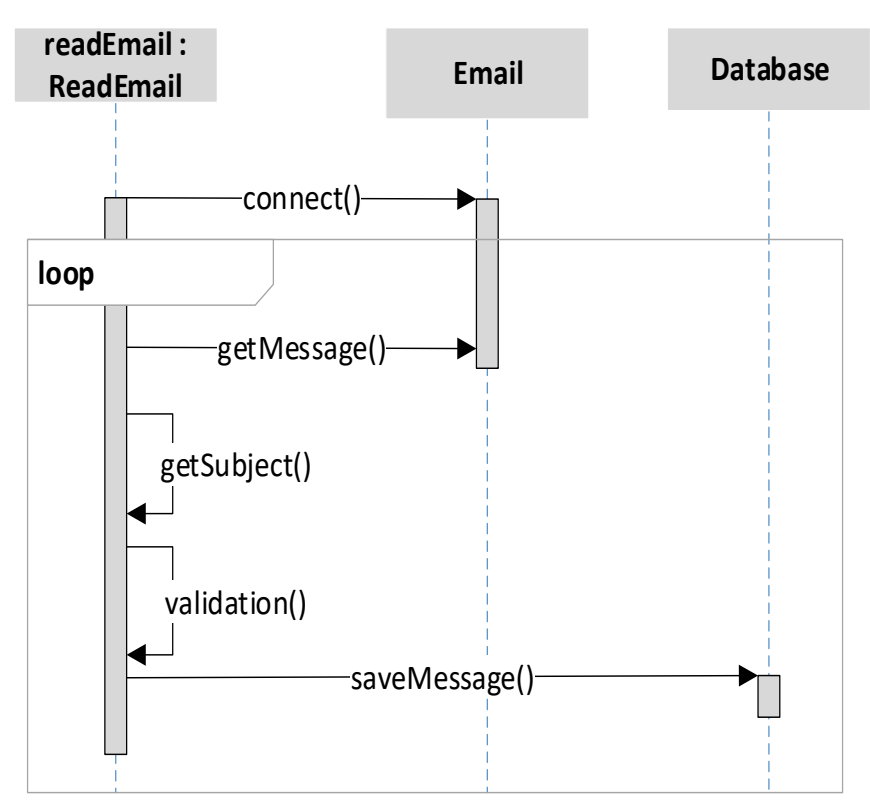

Fig 5. Sequence Diagram of Email Auto Inbox Checking.

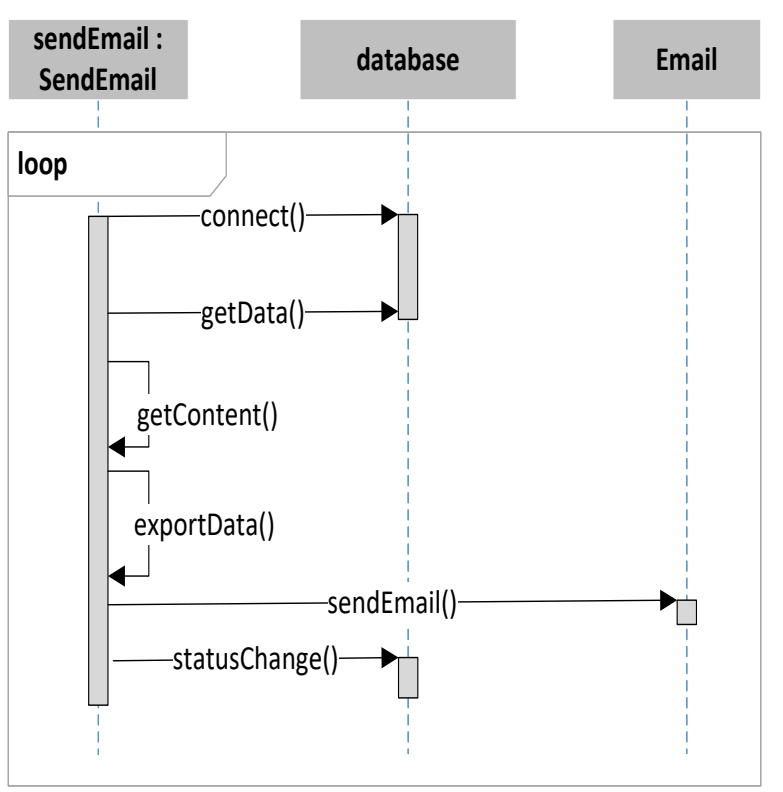

Fig 6. Sequence Diagram of Email Auto Delivery. 


\section{c) Sequence Diagram of Email Auto-reply Application}

The next step is to create a sequence of diagram to illustrate the logical flow of the developed application. Sequence diagram of email auto-reply application can be seen in Fig. 5 and 6 .

\section{d) Format Email Designing to Information Request of} Lecture Schedule

To get the schedule of lectures, lecturers, students and laboratory staff should send an email to the specified format. Here is the design format of the email to request lecture schedule information:

1) Email Subject must be filled with the word JADWAL

2) Format of Email Format Guidelines Request PANDUAN

3) Format of Lecture Schedule Request JADWAL\#SEMESTER\#LEVEL Example: JADWAL\#3\#S1

4) Format of Schedule for Middle Exams (UTS) Request UTS\#SEMESTER\#LEVEL, Example: UTS\#5\#S1

5) Format of Schedule for Final Exams (UAS) Request UAS\#SEMESTER\#LEVEL Example: UAS\#7\#S1

6) Format of Minutes of Class Request (BAP) BAP\#LECTURERCODE Example: BAP\#SYAHRUL

7) Format of Class Use Schedule Request RUANG \# CLASSNAME

Example: RUANG\#5204

8) Format of Laboratory Schedule Request

LAB \# LABNAME

Example: LAB\#LAB4

9) Format OF Lecturer Phone Number Request TELEPON\#LECTURERCODE Example: TELEPON\#SYAHRUL

e) Email Format Design of Reports Inquiry on Email Entry and Email Delivery Data.

To get email reports of email entry data and email delivery, secretary should send email to the specified format. Here is the design of the email format for information requests on the report data:

1) Subject of the email must be filled with the word JADWAL

2) The format of report requests

LAP\# START DATE-END DATE

Example: LAP\#30/12/2018-31/12/2018

\section{Database Design}

Email auto-reply application requires a database to store email data in accordance with a format which is determined. As for the number of tables which are needed for this application is two tables as follows in Fig. 7.

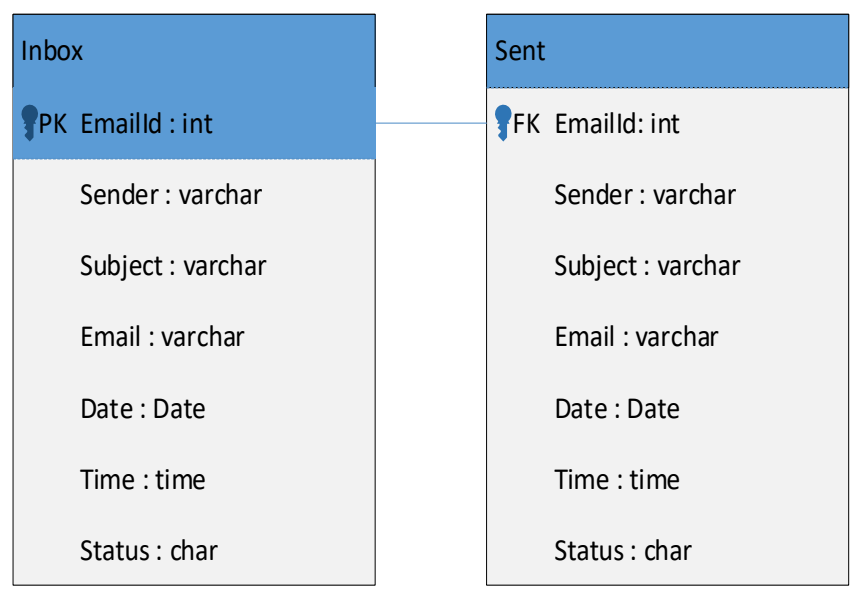

Fig 7. Database Design.

\section{f) Applications Designing of Email Auto-reply}

Email auto-reply application consists of two forms: one form to read incoming emails and then save the email data to the database and another for reading email format and then sends the schedule information in accordance with the request format. Both of the forms can be seen in Fig. 8 and 9.

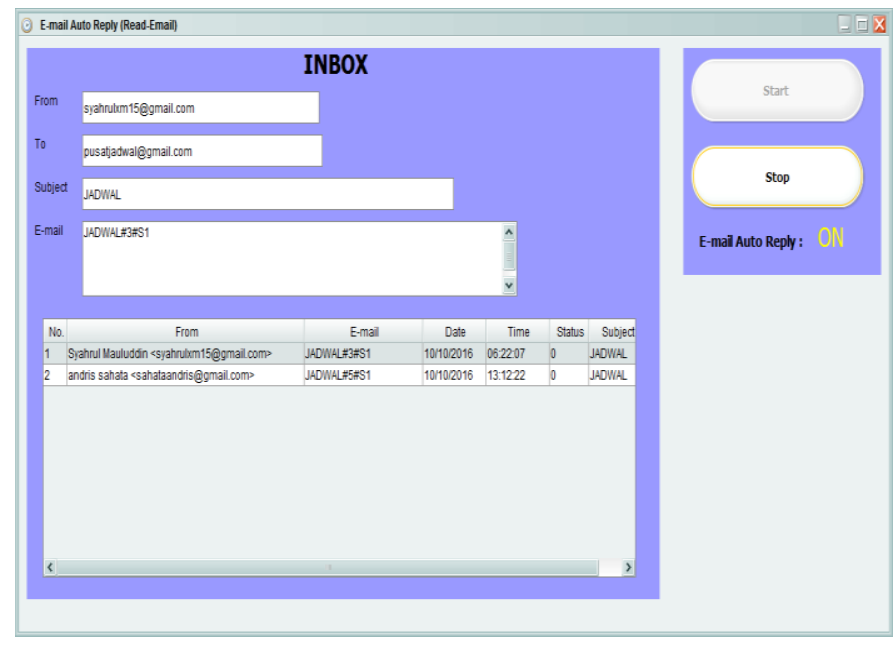

Fig 8. Form of Email Auto Inbox Checking.

As for the algorithm design of the email auto inbox checking, it can be explained descriptively as follows:

Step 1: Click the start button to activate the timer

Step 2: Connection to email and inbox folder

Step 3: Every five seconds, take and check the number of emails in inbox

Step 4: If the number of emails is now larger than the number of previous emails then read the last email

Step 5: Check the last email subject if the format is appropriate then save it to database

Step 6: Do repetition of step 3 to 5 


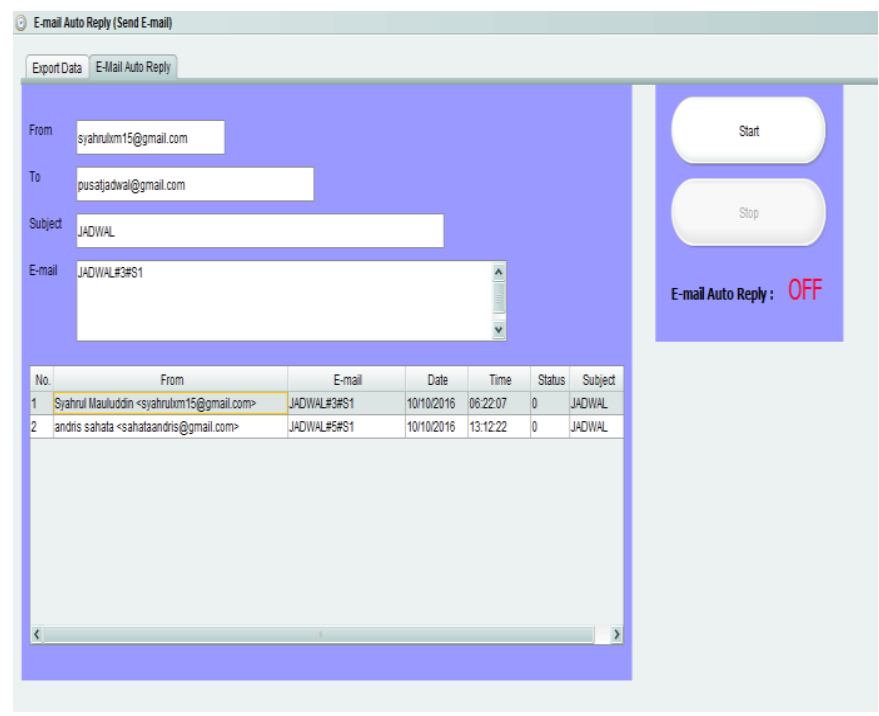

Fig 9. Form of Email Auto Delivery.

As for the algorithms design of email auto delivery, it can be explained descriptively as follows:

Step 1: Click the start button to activate the timer

Step 2: Every five seconds do connection to database, show new email data from database to table component

Step 3: Take email data on the first line, take emails contents on email column

\section{Step 4: Split or extract Email format}

Step 5: Run method of schedule data export in accordance with email format

Step 6: Send email with attachments

Step 7: Change email data status in database into already sent.

Step 8: Do repetitions of step 2 to 7

\section{CONCLUSION}

With the email auto-reply application, it is expected to lecturers, students and laboratory staff can easily obtain lecture schedule information. Then they can get the latest lecture schedule information from the same source so that there is no difference in class schedules between them.

\section{ACKNOWLEDGMENT}

This research project is supported by Directorate of Research and Community Service, Ministry of Research, Technology and Higher Education of Indonesia in 2018 Fiscal Year.

\section{REFERENCES}

[1] Daryanto, Keterampilan Dasar Pengoprasian Komputer. (Basic Skills of Computer Operating). Bandung: Yrama Widya, 2004.

[2] A. S. Hidayat, "Perancangan Mail Server Intranet Berbasis Web Base Dengan Optimalisasi Operasi Sistem Client. (Web-Base Based Intranet Mail Server Designing with System Client Operating Optimization)," J. Tek. Komput., vol. 1, no. 1, hal. 1-10, 2015.

[3] P. Heinlein dan P. Hartleben, The Book of IMAP: Building a Mail server with Courier and Cyrus. Munich: Press GmbH, 2008.

[4] T. M. Zakaria dan O. Wongso, "Studi dan Implementasi Teknologi Flashdisk dan Email Gateway dalam Penyewaan Alat pada Perusahaan X (The Study and Implementation on Technology of Flashdisk and Email Gateway in X Equipment Rental Company)," J. Inform., vol. 6, no. 2, hal. 161-174, 2012.

[5] M. Fowler, UML Distilled 3rd Edition. Yogyakarta: Andi, 2005.

[6] M. Grgec dan R. Mužar, "Role of UML Sequence Diagram Constructs in Object Lifecycle Concept," J. Inf. Organ. Sci., vol. 31, no. 1, hal. 63-74, 2007.

[7] F. Tomhart, Afriyudi, dan M. Bakti, "Optimasi Penjadwalan Perkuliahan Di Universitas Tridinanti Palembang”. Jurnal Ilmiah Teknik Informatika Ilmu Komputer (Lectures Scheduling Optimization at the University of Tridinanti Palembang," J. Ilm. Tek. Inform. Ilmu Komput., vol. 11, no. 2, hal. 1-11, 2013.

[8] E. K. Kendall dan E. J. Kendall, Systems Analysis and Design, 7th ed. New Jersey: Pearson Prentice Hall, 2008.

[9] I. Ikbal dan S. Mauluddin, "Classroom Booking Information System Integrated with Course Scheduling Information System," in IOP Conference Series: Materials Science and Engineering, 2018, vol. 407, no. 1. 